\title{
Assessing the profitability of intraday opening range breakout strategies*
}

\author{
Ulf Holmberg, Carl Lönnbark, Christian Lundström \\ Department of Economics \\ Umeå School of Business and Economics \\ Umeå University \\ SE-901 87 Umeå
}

\begin{abstract}
Is it possible to beat the market by mechanical trading rules based on historical and publicly known information? Such rules have long been used by investors and in this paper, we test the success rate of trades and profitability of the Open Range Breakout (ORB) strategy. An investor that trades on the ORB strategy seeks to identify large intraday price movements and trades only when the price moves beyond some predetermined threshold. We present an ORB strategy based on normally distributed returns to identify such days and find that our ORB trading strategy result in significantly higher returns than zero as well as an increased success rate in relation to a fair game. The characteristics of such an approach over conventional statistical tests is that it involves the joint distribution of Low, High, Open and Close over a given time horizon.
\end{abstract}

Keywords: Bootstrap, Crude oil futures, Contraction-Expansion principle, Efficient market hypothesis, Martingales, Technical Analysis.

JEL classification: C49, G11, G14, G17.

\footnotetext{
${ }^{*}$ The second author gratefully acknowledges the financial support from the Wallander foundation. We thank Kurt Brännäs and Tomas Sjögren for insightful comments and suggestions.
} 


\section{Introduction}

The Efficient Market Hypothesis (EMH) of Fama $(1965,1970)$ asserts that current asset prices fully reflect available information (see also Fama, 1991) implying that asset prices evolve as random walks in time. Consequently, tests of the EMH have traditionally been designed to catch deviations from random walk prices and in the massive literature on the subject one is bound to find support for both acceptances and rejections of the hypothesis (e.g., Malkiel, 1996; Lo, 2001). In particular, an assertion of the EMH is that it should not be possible to base a trading strategy on historical prices (so-called filter rules or technical trading) and earn positive expected returns. However, the fact remains that the use of filter rules is a widespread phenomenon. Barclay Hedge estimates that filter based Hedge Funds within the Managed Futures category manage over 300 Billion USD in 2011 and is today the largest hedge fund category with respect to assets under management. Indeed, some filter rule traders appear to consistently outperform the market (see Schwager, 1989, for a classic reference) and the subject has been given due attention in the literature (e.g. Brock, Lakonishok, and LeBaron, 1992; Gençay, 1996, 1998). Testing of the profitability of trading rules has traditionally been carried out based on a (at least) daily investment horizon. However, as discussed in Taylor and Allen (1992) the use of filter rules among practitioners appears to increase with the frequency of trading (see also Schulmeister, 2009). In particular, many strategies are typically employed intraday and to assess their potential profitability one would typically require intraday data. The relative unavailability of intraday data may thus be a possible explanation for the apparent lagging behind of the research community.

In this paper we remove this obstacle and propose a quite novel approach on how to assess the profitability when only records of daily high, low, opening and close are available. Obviously, there is a plethora of filter rules out there and the one we have in mind in the present paper is the so-called opening range breakout (ORB), which is typically adopted intraday. This rule is based on the premise that if the market moves a certain percentage from the opening price level, the odds favor a continuation of that move. An ORB filter suggests that, long (short) positions are established at some predetermined price threshold a certain percentage above (below) the opening price. 
To evoke the testing strategy and gain intuition on the way we first note that the rationale behind using an ORB filter is the believe in so-called momentum in prices (e.g. Jegadeesh and Titman, 1993). That is, the tendency for rising asset prices to rise further and falling prices to keep falling. In the behavioral finance literature the appearance of momentum is often attributed to cognitive biases from irrational investors such as investor herding, investor over- and under reaction, and confirmation bias (see Barberis et al., 1998; Daniel et al., 1998). However, as discussed in Crombez (2001) momentum can also be observed with perfectly rational traders. In pioneering the ORB strategy Crabel (1990) presented the so-called Contraction-Expansion (C-E) principle. The principle asserts that markets alternates between regimes of contraction and expansion, or, periods of modest and large price movements, respectively. An ORB strategy may be viewed as a strategy of identifying and profiting from days of expansion. In passing we note the resemblance with the stylized fact of volatility clustering in financial return series (e.g. Engle, 1982).

Now, a seemingly quite reasonable assumption is that markets for the most part are relatively efficient with prices evolving as random walks in time, or equivalently, returns are martingales. Thus, a heuristic use of the law of large number implies normally distributed returns. According to the (C-E) principle these calm days could be considered as periods of contraction during which the returns are normally distributed. Now, during periods of expansions traders activates ORB strategies and the profitability of them implies that the martingale property breaks down with non-normality as a consequence. Building on this reasoning our testing strategy is simply based on identifying days of large intraday movements and evaluating the expected return on these days. In particular, if on a given day the price threshold implied by the rule is above (below) the high (low) price we deduce that a long (short) position was established at some point during this day. To assess statistical significance we build on Brock et al. (1992) and use a bootstrap approach adapted to the present case.

The remainder of the paper is organized as follows. In Section 2 we briefly review the underlying theory and give an account of the ORB strategy. In this section we also outline our proposed test for profitability. Section 3 gives results for the empirical application and the fourth section concludes. 


\section{Martingale prices and momentum based trading strategies}

We denote by $P_{t}^{o}, P_{t}^{h}, P_{t}^{l}$ and $P_{t}^{c}$ the opening, high, low and, closing price on day $t$, respectively. A point in time on day $t$ is given by $t+\delta, 0 \leq \delta \leq 1$. Note that $P_{t}^{o}=P_{t}$ and $P_{t}^{c}=P_{t+1}$. The set

$\Psi_{t+\delta}$ contains the information available at time $t+\delta$. Furthermore, let $\psi^{u}\left(\psi^{l}\right)$ denote a certain threshold price level that is such that if the price crosses it from below (above) a momentum investor acts, i.e. takes a long (short) position. For ORB investors, these threshold price are often set in terms of some pre-determined (large) relative change, $\rho$, from the opening price such that $\psi_{t}^{u}=(1+\rho) P_{t}^{o}$ and $\psi_{t}^{l}=(1-\rho) P_{t}^{o}$. For the purpose of this paper we assume that all positions are closed at the end of the trading day. Hence, no type of money management techniques such as a stop loss, trailing loss, profit stop are considered.

Within the context of the present paper it is natural to involve the martingale pricing model (MPT) of Samuelson (1965). If capital markets are efficient with respect to $\Psi_{t+\delta}$ some prescribed formula based on $\Psi_{t+\delta}$ should not result in systematic success implying that prices are martingales with respect to this information set. In particular,

$$
E\left[P_{t}^{c} \mid \Psi_{t+\delta}\right]=P_{t+\delta}
$$

A direct consequence of martingale pricing is that any investment should earn a zero expected return

$$
E\left[R_{t+\delta}^{c} \mid \Psi_{t+\delta}\right]=0,
$$

where $R_{t+\delta}^{c}=\log \left(P_{t}^{c} / P_{t+\delta}\right)$. As such, any investment within the MPT framework is a "fair game" and from the martingale central limit theorem it follows that the returns are normally distributed (Brown, 1971).

Now, momentum investments are based on the premise that, if the market moves a certain percentage from the opening price level, the odds favor a continuation of that move. More specifically, a profitable momentum based trading strategy implies that

$$
E\left[P_{t}^{c} \mid P_{t+\delta}>\psi_{t}^{u}\right]>P_{t+\delta} \text { and/or } E\left[P_{t}^{c} \mid P_{t+\delta}<\psi_{t}^{l}\right]<P_{t+\delta} .
$$




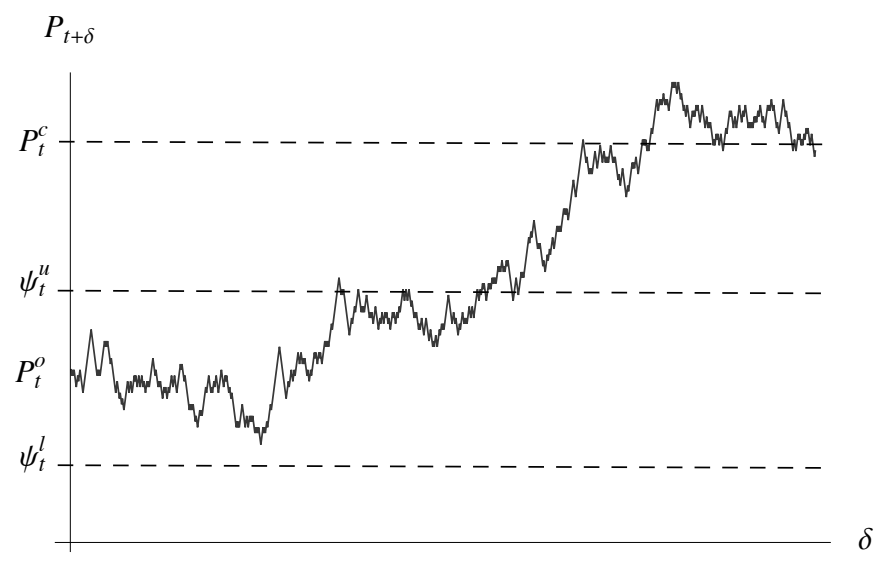

Figure 1: An ORB strategy trader enters a long position if the intraday price exceeds $\psi_{t}^{u}$.

As such, the breaking down of the martingale property implies that the martingale central limit theorem no longer applies. Thus, it is natural to define $\rho$ as a daily return that is unlikely to occur given normally distributed returns

$$
\rho_{\alpha}=\hat{\mu}+\hat{\sigma} q_{\alpha}
$$

where $\hat{\mu}$ and $\hat{\sigma}$ are estimates of the mean and standard deviation of $R_{t}^{c}=\log \left(P_{t}^{c} / P_{t}^{o}\right)$, respectively, and $q_{\alpha}$ the inverse of the standard normal cumulative distribution function evaluated at $\alpha$. Figure 1 illustrates a profitable intraday trade based an ORB strategy. The price opens at $P_{t}^{o}$ and as long as the price stays within "normal bounds", i.e. within $\left(\psi_{t}^{u}, \psi_{t}^{l}\right)$, the trader refrains from action but as soon as $P_{t+\delta}=\psi_{t}^{u}$, the trader initiates a long position, anticipating a continuation of the price moving in the same direction.

Given that an ORB strategy is based on intraday price movements, as illustrated in Figure 1 , it is clear that a perfect test of profitability requires information on the intraday price paths. The challenge we take on here is that of designing a test with access only to records of daily opening, high, low and closing prices. Our basic observation is that if the daily high (low) is higher (lower) than the set $\psi_{t}^{u}\left(\psi_{t}^{l}\right)$, we know with certainty that a buy (sell) signal was triggered at some point during the day and that a position was initiated at $\psi_{t}^{u}\left(\psi_{t}^{l}\right)$. For the purpose of this paper we assume a perfect order fill at the threshold price, a zero bid ask spread, as well as 
zero commissions. Consequently, real-life trading produce slightly different results.

Upon defining the return series $R_{t}^{\operatorname{long}}=\log \left(P_{t}^{c} / \psi_{t}^{u}\right)$ and $R_{t}^{\text {short }}=\log \left(P_{t}^{c} / \psi_{t}^{l}\right)$ we may consider the averages

$$
\begin{aligned}
\bar{R}^{\text {long }} & =\frac{\sum \mathbf{1}\left(P_{t}^{h}>\psi^{u}\right) R_{t}^{\text {long }}}{\sum \mathbf{1}\left(P_{t}^{h}>\psi^{u}\right)}, \\
\bar{R}^{\text {short }} & =-\frac{\sum \mathbf{1}\left(P_{t}^{l}<\psi^{l}\right) R_{t}^{\text {short }}}{\sum \mathbf{1}\left(P_{t}^{l}<\psi^{l}\right)},
\end{aligned}
$$

where $\mathbf{1}(\cdot)$ is the indicator function. If strategies based on ORB filters are profitable then $\bar{R}^{\text {long }}$ and $\bar{R}^{\text {short }}$ should be significantly larger than zero. To assess statistical significance we rely on the bootstrap approach suggested in Brock et al. (1992). Here, we face additional challenges compared to their work as the case at hand is multivariate with a natural ordering of the level series. A reasonable procedure that accommodates this restriction proceeds as follows.

Assume that the level series share a common trend (cf. co-integration). Hence, considering a "benchmark" series to bootstrap the general levels appears reasonable. The other series may then be obtained as bootstrapped deviations from the benchmark series. To this end we consider the daily opening price as the benchmark series and define $R_{t}^{o}=\log \left(P_{t}^{o} / P_{t-1}^{o}\right), t=2, . ., T$. Also define deviations $R_{t}^{i}=\log \left(P_{t}^{i} / P_{t}^{o}\right)$ for $i=\{h, l, c\}$ and $t=1, . ., T$. Collect these returns in $\mathbf{R}_{t}=\left(R_{t}^{o}, R_{t}^{h}, R_{t}^{l}, R_{t}^{c}\right)$ are then drawn randomly with replacement, generating an pseudo-sample of returns. Based on this sample, an alternative realization of the level series is then generated. This procedure is repeated $N$ times to generate sampling distributions of $\bar{R}^{\text {long }}$ and $\bar{R}^{\text {short }}$ respectively. The sampling distributions are then used in the standard way to test the null of zero expected returns against the alternative of positive ones.

\section{Application}

We apply the testing strategy presented above to a time series of U.S. crude oil futures prices obtained from Commodity Systems Inc covering the period March 30, 1983 to January 26, 2011. When constructing the time series the switch from the near-by contract to the next typically occur around the 20th each month, one month prior to the expiration month (see Pelletier, 1997, 


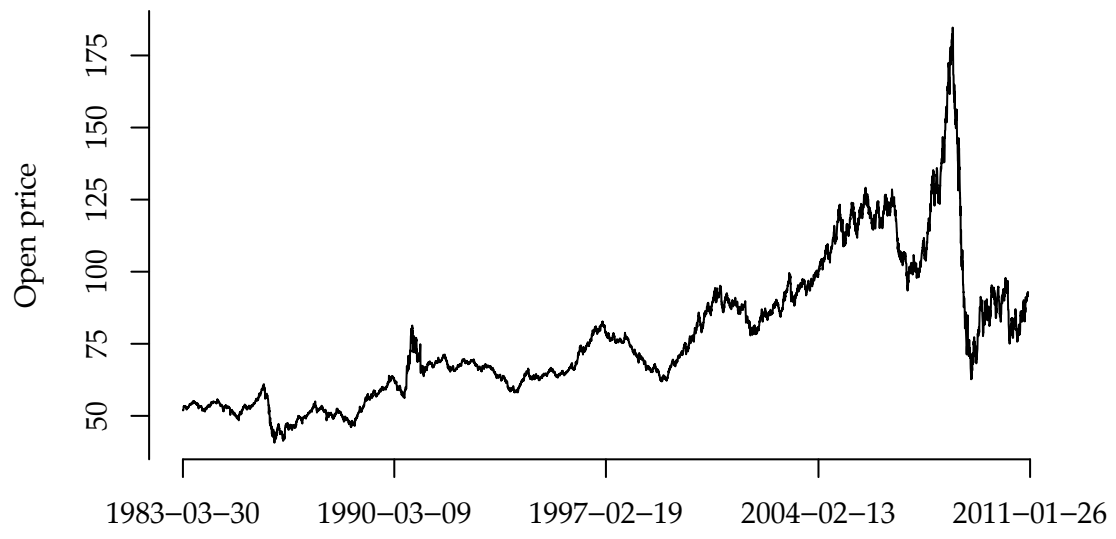

Figure 2: The evolution of the daily open price for U.S. crude oil futures adjusted for roll-over effects from March 30, 1983 to January 26, 2011. Source: Commodity Systems Inc.

Table 1: Descriptives of the daily return series.

\begin{tabular}{cccccccc}
\hline \hline Obs. & Mean & Std.Dev & Min & Max & Skewness & Kurtosis & Jarque-Bera \\
\hline 6976 & 0.02 & 0.72 & -6.06 & 9.90 & 0.16 & 10.26 & 30668
\end{tabular}

for details on the adjustment of roll-over effects). Commodity futures are as easily sold short as bought long, and are not subject to short-selling restrictions while the costs associated with trading (e.g. slippage, bid ask spreads, and commissions) are often relatively low. In Figure 2 we plot the evolution of the level series. The series exhibit a cyclical pattern and follows a positive long run trend reasonably due to inflation. Notable is also the sharp drop during the 2008 sub-prime crisis.

In Table 1 we give some descriptives for the daily returns series, i.e. $R_{t}^{c}$. The series exhibit positive skewness and excess kurtosis and consequently the Jarque-Bera test strongly rejects normality.

The values of the $\rho$ 's (and consequently the treshold prices) are derived from the sample. We thus check ex post for the existence of intraday trending of oil futures prices.

As can be read in Table 2, the ORB strategy results in significant positive average returns suggesting that the "fair game" argument embedded in the Martingale pricing theory does not hold true for adverse price movements. Interestingly, as we tighten the criterion used to determine entry, i.e. if we move further down the tail of a normal distribution, both the success rate and average returns increase. Figure 3 clarifies this relationship. However, it should be 
Table 2: Empirical results. The $\alpha$ is the tail probability, and $\rho$ gives the associated percentage return. $\mathrm{N}$ is the number of trades. freq gives the proportion of trades that result in positive returns, while $\bar{R}$ gives the average returns.

\begin{tabular}{|c|c|c|c|c|c|c|c|c|c|c|c|}
\hline \multirow{7}{*}{ full sample } & \multicolumn{6}{|c|}{ Long } & \multicolumn{5}{|c|}{ Short } \\
\hline & $\alpha$ & $\rho$ & $N$ & freq. & $\bar{R}_{\text {long }}$ & $p$ & $\rho$ & $N$ & freq. & $R_{\text {short }}$ & $p$ \\
\hline & $10 \%$ & 0.9388 & 738 & 0.6057 & 0.2019 & 0.0000 & -0.9013 & 826 & 0.5424 & 0.1439 & 0.0000 \\
\hline & $5 \%$ & 1.1996 & 439 & 0.6036 & 0.2180 & 0.0000 & -1.1621 & 497 & 0.5714 & 0.1784 & 0.0000 \\
\hline & $1 \%$ & 1.6889 & 188 & 0.6117 & 0.2583 & 0.0001 & -1.6513 & 224 & 0.6205 & 0.2442 & 0.0003 \\
\hline & $0.5 \%$ & 1.8680 & 141 & 0.6028 & 0.3108 & 0.0002 & -1.8304 & 172 & 0.6454 & 0.2527 & 0.0008 \\
\hline & $0.1 \%$ & 2.2373 & 80 & 0.7125 & 0.4027 & 0.0010 & -2.1997 & 98 & 0.6225 & 0.2489 & 0.0147 \\
\hline & $\alpha$ & $\rho$ & $N$ & freq. & $\bar{R}_{\text {long }}$ & $p$ & $\rho$ & $N$ & freq. & $\bar{R}_{\text {short }}$ & $p$ \\
\hline & $10 \%$ & 0.7840 & 260 & 0.4923 & 0.0334 & 0.2539 & -0.7574 & 272 & 0.5368 & 0.0871 & 0.0430 \\
\hline 1983-03-30 & $5 \%$ & 1.0024 & 159 & 0.5157 & 0.0711 & 0.1350 & -0.9759 & 156 & 0.5192 & 0.1313 & 0.0401 \\
\hline to & $1 \%$ & 1.4122 & 72 & 0.4861 & 0.1140 & 0.1246 & -1.3857 & 73 & 0.5753 & 0.1978 & 0.0563 \\
\hline 1992-06-29 & $0.5 \%$ & 1.5623 & 57 & 0.4912 & 0.0799 & 0.2467 & -1.5357 & 56 & 0.5893 & 0.2420 & 0.0494 \\
\hline & $0.1 \%$ & 1.8716 & 33 & 0.5758 & 0.1656 & 0.1448 & -1.8451 & 41 & 0.6342 & 0.1026 & 0.2859 \\
\hline & $\alpha$ & $\rho$ & $N$ & freq. & $\bar{R}_{\text {long }}$ & $p$ & $\rho$ & $N$ & freq. & $\bar{R}_{\text {short }}$ & $p$ \\
\hline & $10 \%$ & 0.6069 & 373 & 0.5657 & 0.0374 & 0.0357 & -0.5947 & 371 & 0.5148 & 0.0307 & 0.0734 \\
\hline 1992-06-30 & $5 \%$ & 0.7772 & 195 & 0.5795 & 0.0634 & 0.0196 & -0.7650 & 214 & 0.5327 & 0.0228 & 0.2172 \\
\hline to & $1 \%$ & 1.0966 & 62 & 0.5807 & 0.0843 & 0.0357 & -1.0845 & 79 & 0.5317 & -0.0258 & 0.6814 \\
\hline $2001-10-11$ & $0.5 \%$ & 1.2136 & 53 & 0.3962 & 0.0068 & 0.4546 & -1.2015 & 57 & 0.5790 & -0.0608 & 0.8091 \\
\hline & $0.1 \%$ & 1.4548 & 20 & 0.5000 & 0.0254 & 0.4061 & -1.4426 & 27 & 0.3333 & -0.0290 & 0.6420 \\
\hline & $\alpha$ & $\rho$ & $N$ & freq. & $\bar{R}_{\text {long }}$ & $p$ & $\rho$ & $N$ & freq. & $\bar{R}_{\text {short }}$ & $p$ \\
\hline & $10 \%$ & 1.2956 & 245 & 0.6612 & 0.2813 & 0.0000 & -1.2216 & 300 & 0.5967 & 0.2483 & 0.0000 \\
\hline 2001-10-12 & $5 \%$ & 1.6524 & 138 & 0.6522 & 0.3405 & 0.0004 & -1.5784 & 177 & 0.6328 & 0.2734 & 0.0001 \\
\hline to & $1 \%$ & 2.3216 & 50 & 0.8000 & 0.5155 & 0.0063 & -2.2477 & 64 & 0.6406 & 0.3879 & 0.0006 \\
\hline 2011-01-26 & $0.5 \%$ & 2.5667 & 44 & 0.7500 & 0.4926 & 0.0062 & -2.4927 & 48 & 0.6667 & 0.3892 & 0.0008 \\
\hline & $0.1 \%$ & 3.0718 & 23 & 0.8261 & 0.6397 & 0.0096 & -2.9979 & 28 & 0.7143 & 0.3763 & 0.0054 \\
\hline
\end{tabular}

noted that by moving down the tail of the normal distribution, we also reduce the number of trades, reducing the investors potential profits.

Dividing the full data set into three sub-samples, 1983-03-30 to 1992-06-29, 1992-06-30 to 2001-10-11, and finally 2001-10-12 to 2011-01-26 we find that the most recent time period drives the result. Given the possible resemblance between the ORB strategy and the stylized fact of volatility clustering in financial returns series, one plausible explanation is the relatively high volatility in the 2001-10-12 to 2011-01-26 period. After all, ORB is a directional strategy in the sense that either a long or a short position is established and hence it is basically long volatility in contrast to hedge fund strategies such as Long Short Equity, Market Neutral strategies or different variants of Arbitrage strategies to mention a few. Market volatility and ORB profitability should be expected to go hand in hand. 


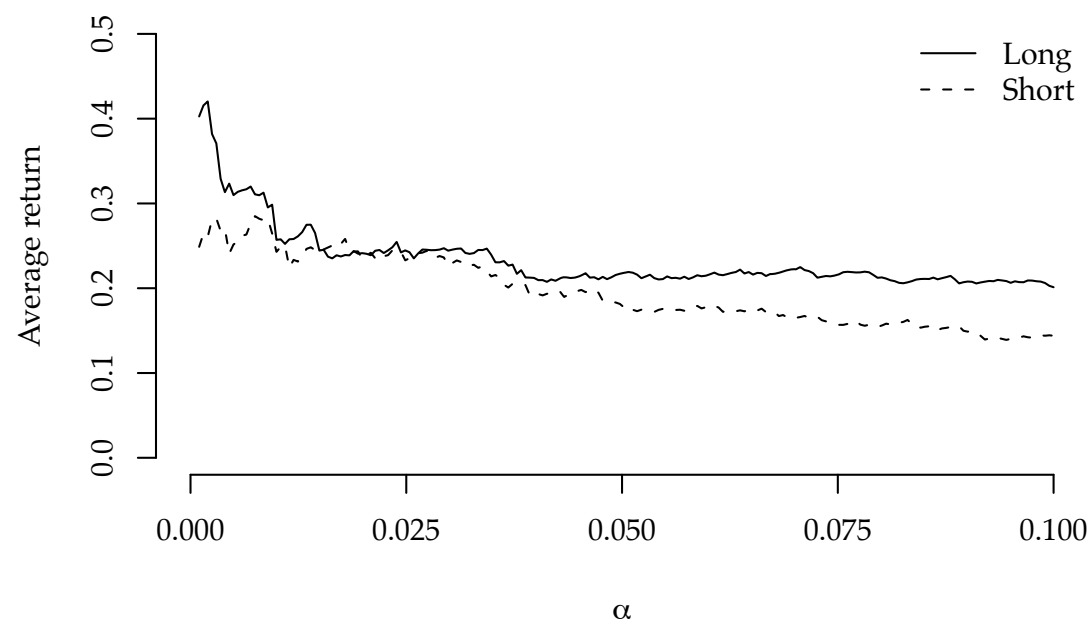

Figure 3: Average returns on the tail probability $(\alpha)$.

\section{Concluding discussion}

We proposed a way of assessing the profitability of intraday ORB strategies when long records of daily opening, high, low and closing prices are available. In an empirical application we employ our testing strategy to U.S. crude oil futures. Using the full sample we find a remarkable success of the of ORB strategies. However, splitting up the full sample into three sub-periods reveals that this finding is not robust to time and to a large extent explained by the most recent (and most volatile) period. In this sense, our results relate to the findings in Gençay (1998), that mechanical trading rules tend to result in higher profits when markets "trend" or in times of high volatility.

A point to note is that our testing strategy will underestimate the actual profits since the closing of the positions is assumed to occur at the daily close. Thus, days when the momentum does not carry through to the end of the day or even reverses intraday will be included. In practice, the losses on these days will be limited by so-called stop losses.

Notable is also the our filter results in relatively few trades, which restricts potential profits. Most likely though the orb trader simultaneously monitors and acts on several markets.

Admittedly, transaction costs in terms of commission fees and bid-ask spreads will consume some of the profits. However, for the market under consideration these are relatively small. A reasonable estimate is $0.04 \%$, or $0.08 \%$ round trip. 


\section{References}

Barberis, N., A. Shleifer, And R. Vishny (1998): "A Model of Investor Sentiment," Journal of Financial Economics, 49, 307-343.

Brock, W., J. LAKonishok, And B. LeBaron (1992): "Simple Technical Trading Rules and the Stochastic Properties of Stock Returns," Journal of Finance, 47, 1731-64.

Brown, B. M. (1971): "Martingale Central Limit Theorems," The Annals of Mathematical Statistics, 42, 59-66.

Crabel, T. (1990): Day Trading With Short Term Price Patterns Day Trading With Short Term Price Patterns and Opening Range Breakout, Greenville, S.C. : Traders Press.

Crombez, J. (2001): "Momentum, Rational Agents and Efficient Markets," Journal of Psychology and Financial Markets, 2, 190-200.

Daniel, K., D. Hirshleifer, and A. Subrahmanyam (1998): "Investor Psychology and Security Market Under- and Overreactions,," Journal of Finance, 53, 1839-1885.

Engle, R. F. (1982): "Autoregressive Conditional Heteroscedasticity with Estimates of the Variance of United Kingdom Inflation," Econometrica, 50.

FAmA, E. (1965): "The Behavior of Stock Market Prices," Journal of Business, 38, 34-105. (1970): "Efficient Capital Markets: A Review of Theory and Empirical Work," The Journal of Finance, 25, 383-417.

_ (1991): "Efficient Capital Markets: II," Journal of Finance, 46, 1575-617.

GençAy, R. (1996): "Non-linear Prediction of Security Returns with Moving Average Rules," Journal of Forecasting, 15, 165-174.

_ (1998): "The Predictability of Security Returns with Simple Technical Trading Rules," Journal of Empirical Finance, 5, 347-359.

Jegadeesh, N. And S. Titman (1993): "Returns to Buying Winners and Selling Losers: Implications for Stock Market Efficiency," Journal of Finance, 48, 65-91. 
Lo, A. (2001): A Non-random Walk Down Wall Street, Princeton Paperbacks.

Malkiel, B. G. (1996): A Random Walk Down Wall Street, W. W. Norton.

Pelletier, B. (1997): "Computed Contracts: Their Meaning, Purpose and Application," CSI Technical Journal, 13, 1-6.

Samuelson, P. A. (1965): "Proof That Properly Anticipated Prices Fluctuate Randomly," Industrial Management Review, 6, 41-49.

Schulmeister, S. (2009): "Profitability of technical stock trading: Has it moved from daily to intraday data?" Review of Financial Economics, 18, 190-201.

Schwager, J. D. (1989): Market Wizards: Interviews with Top Traders, HarperCollins publishers Inc.

Taylor, M. P. And H. Allen (1992): "The Use of Technical Analysis in the Foreign Exchange Market," Journal of International Money and Finance, 11, 304-314. 